\title{
Evaluación del antagonismo de Trichoderma sp. y Bacillus subtilis contra tres patógenos del ajo
}

\author{
Evaluation of antagonism of Trichoderma sp. and \\ Bacillus subtilis against three garlic pathogens
}

Karina Astorga-Quirós'

Karla Meneses-Montero ${ }^{2}$

Claudia Zúniga-Vega ${ }^{3}$

Jaime Brenes-Madriz ${ }^{4}$

William Rivera-Méndez ${ }^{5}$

Fecha de recepción: 07 de octubre del 2013

Fecha de aprobación: 21 de diciembre del 2013

Astorga-Quirós, K; Meneses-Montero, K; Zúñiga-

Vega, C; Brenes-Madriz, j; Rivera-Méndez, W.

Evaluación del antagonismo de Trichoderma sp.

y Bacillus subtilis contra tres patógenos del ajo.

Tecnología en Marcha. Vol. 27, Nº 2. Pág 82-91

Estudiante de Ingeniería en Biotecnología. Instituto Tecnológico de Costa Rica. Costa Rica. Correo electrónico: kariasq@yahoo.com.

2 Investigadora. Escuela de Biología. Instituto Tecnológico de Costa Rica. Costa Rica. Correo electrónico: kmeneses@itcr.ac.cr.

3 Académica. Escuela de Biología. Instituto Tecnológico de Costa Rica. Costa Rica. Correo electrónico: czuniga@itcr.ac.cr.

4 Académico. Escuela de Biología. Instituto Tecnológico de Costa Rica. Costa Rica. Correo electrónico: jabrenes@itcrac.cr.

5 Académico. Escuela de Biología. Instituto Tecnológico de Costa Rica. Costa Rica. Correo electrónico: wrivera@itcr.ac.cr. 


\section{Palabras clave}

Allium sativum; Bacillus subtilis; Trichoderma sp:; Sclerotium cepivorum; Pseudomonas marginalis; Penicillium sp.

\section{Resumen}

La producción y calidad del cultivo del ajo criollo (Allium sativum) se ven limitadas por diversas enfermedades de origen fungoso y bacterial, que llevan al productor a aplicar estrategias de control químico y en algunos casos abandonar la actividad por un incremento en las pérdidas. El control biológico es una estrategia útil para combatir este tipo de microorganismos. El objetivo de esta investigación consistió en evaluar el antagonismo in vitro de Trichoderma sp. y Bacillus subtilis contra tres de los principales patógenos del ajo: Sclerotium cepivorum, Penicillium sp. y Pseudomonas marginalis. Las especies mencionadas se aislaron e identificaron con pruebas bioquímicas y claves taxonómicas respectivamente y se determinó su actividad antagónica y efecto inhibitorio utilizando el crecimiento en platos duales. La cepa de B. subtilis mostró un potencial con valores bajos de PICR: 14,087 ante S. cepivorum y 3,328 ante Penicillium sp., por lo que se clasifica como un mal biocontrolador. Por su parte, Trichoderma presentó un potencial muy alto, con valores de PICR de 40,2 10 frente a S. cepivorum y de 45,034 ante Penicillium sp., lo que indica que es un muy buen controlador. Los resultados apoyan el potencial de las cepas de Trichoderma sp. como agentes de control biológico frente a la pudrición causada por Penicillium del ajo, la bacteriosis por P. marginalis y la pudrición blanca por S. cepivorum. No así Bacillus subtilis, pues la cepa aislada demostró poco potencial como biocontrolador.

\section{Key words}

Allium sativum; Bacillus subtilis; Trichoderma sp.; Sclerotium cepivorum; Pseudomonas marginalis; Penicillium sp.

\begin{abstract}
The production and quality of the cultivate garlic field (Allium sativum) is limited by diverse fungal diseases and bacteria that lead the producer to implement the application of chemical control strategies and in some cases the abandonment of the activity by an increase in losses. Biological control is a useful strategy to combat these types of microorganisms. The objective of this research was to evaluate the in vitro antagonism of the Trichoderma sp. and Bacillus subtilis against three major pathogens of garlic: Sclerotium cepivorum, Penicillium sp. and Pseudomonas marginalis. The species mentioned were isolated and identified by biochemical and taxonomic keys respectively and tested for antagonistic activity and inhibitory effect on growth using dual plates. Strain B. subtilis showed a low potential with low values of PICR, 14,087 against S. cepivorum and 3,328 against Penicillium sp., this is bad biocontrol. Regarding Trichoderma sp, it presented a very high potential, with values of 40,2 I 0 PICR against S. cepivorum and 45,034 against Penicillium sp. The valuation classifies it as a very good biocontrol. The results obtained in these studies support the potential of the strains of Trichoderma sp as biological control agents against Penicillium sp garlic rot, P. marginalis bacteria and white rot by $S$. cepivorum. This is not the case with $B$. subtilis, as the isolate proved otherwise.
\end{abstract}




\section{Introducción}

En Costa Rica, la producción y la calidad del cultivo del ajo (Allium sativum L.) se ven limitadas por diversas enfermedades de origen fungoso, bacterial y viral, que inciden en la baja productividad y en una reducción de la superficie sembrada. Esto, sumado a las políticas arancelarias que reducen o anulan los impuestos a los productos importados, ha causado un incremento de las importaciones desde China y otros países que estancan o dejan rezagada, aún más, la producción del ajo criollo (Brenes et al. 2013, Guillén et al. 201।, Rivera et al., 2012).

Las enfermedades más comunes de esta hortaliza afectan tanto al follaje como a los órganos subterráneos, principalmente por la podredumbre de los bulbos causado por especies de Penicillium, podredumbre blanda en bulbos y sistema radicular por Sclerotium cepivorum Berk o bacteriosis ocasionadas por Pseudomonas marginalis (Lisboa, 2003, Rojas et al., 2009). En el caso de S. cepivorum, la gran capacidad de supervivencia de los esclerocios, que pueden permanecer en el suelo por hasta 20 años, dificulta aún más el manejo (Velásquez y Medina, 2004, Pinto et al., 1998).

Por su parte, el control biológico de plagas, una de las alternativas que existe, debe basarse en técnicas preventivas, fomentando el desarrollo óptimo de las plantas y su resistencia natural a plagas y enfermedades. Los microorganismos más usados en ajo incluyen Streptomyces, Pseudomonas, Agrobacterium, Trichoderma y Bacillus (Reinoso et al., 2006, Izzeddin y Medina, 20 I I). En el caso de Trichoderma, la especie T. harzianum ejerce su actividad de hiperparásito competitivo con la producción de metabolitos antifúngicos y enzimas hidrolíticas, que provocan cambios estructurales a nivel celular, sin atacar a las plantas superiores; mientras que las cepas de B. subtilis se caracterizan por producir antibióticos y otras sustancias con capacidad antifúngica que impiden el establecimiento de los patógenos (Ezziyyani et al., 2004b).

Tanto T. harzianum como B. subtilis crecen rápidamente en cultivo líquido, forman endosporas y producen metabolitos secundarios que reducen la posibilidad de desarrollar resistencia por parte del patógeno. También presentan la ventaja de que se degradan de forma paulatina. El grado de ataque de estos antagonistas depende de factores como el ambiente, el estado del tejido vegetal $y$, en el caso de los hongos, de la cantidad y calidad del inóculo (Hernández et al. 2007). Varios autores han analizado la liberación de compuestos con propiedades antifúngicas como la subtilina y otros antibióticos de la familia de las iturinas. Se ha demostrado que $B$. subtilis no solo produce compuestos antibacteriales sino también antifúngicos (Santander, 20I2).

El objetivo de esta investigación consistió en estudiar la capacidad antagónica in vitro del hongo Trichoderma sp. y la bacteria B. subtilis frente a tres patógenos del ajo: $S$. cepivorum, P. marginalis y Penicillium sp., para su utilización como una estrategia futura para lograr un control más efectivo de estas enfermedades en el campo.

\section{Materiales y métodos}

\section{Localización y material experimental}

Las pruebas y procedimientos se llevaron a cabo durante el segundo semestre de 2012 y 2013 , en las instalaciones del Centro de Investigación en Biotecnología (CIB) y los laboratorios de la Escuela de Biología del Instituto Tecnológico de Costa Rica ITCR).

Para el bioensayo se emplearon muestras de tejido vegetal (bulbos de ajo blanco), rizosfera y suelo con signos de las diferentes enfermedades en el campo, procedentes de la zona norte de Cartago, Costa Rica.

Aislamiento y caracterización de Penicillium sp., Sclerotium cepivorum, Pseudomonas marginalis, Bacillus subtilis y Trichoderma sp.

El aislamiento y caracterización de Penicillium sp., S. cepivorum y P. marginalis se realizó según la metodología utilizada por Astorga et al. (en prensa).

Para B. subtilis se utilizó el método descrito por Reyes et al. (201 I). Se pesó I g de cada muestra de suelo y se suspendió en $9 \mathrm{ml}$ de agua destilada estéril en un tubo de centrífuga de fondo cónico de $15 \mathrm{ml}$. Se homogeneizó vigorosamente en un vórtex durante 3-5 min. Los tubos se colocaron a $80^{\circ} \mathrm{C}$ por 15 min y se sembraron $200 \mu$ para una dilución final de 1:200 en placa Petri con medio agar nutritivo. Finalmente, se incubó a $28 \pm 2^{\circ} \mathrm{C}$ durante $24 \mathrm{~h}$. Se seleccionaron colonias según su forma, tamaño, bordes, elevación, color y textura con base en lo descrito por Claus y Berkeley (1986). 
Se resembraron en forma individual en agar nutritivo, con 5 repeticiones de la muestra inicial. Se resembraron en medio agar sangre y se volvieron a cultivar en agar glucosado al $2 \%$. A cada colonia se le realizó una caracterización bioquímica (Bergey, 2005). Se aplicaron las siguientes pruebas: oxidasa, catalasa, crecimiento en $\mathrm{NaCl}$ al 6,5\%, asimilación de citrato, Voges-Proskauer, (producción de acetoína o 2,3 butanodiol), reducción de nitratos, almidón y rojo de metilo. Con la tinción de Gram, se determinó el tipo de pared y el tipo de agregación, mediante observación microscópica a 1000x.

Para los cultivos de Trichoderma sp. se activaron cepas conservadas a $-10^{\circ} \mathrm{C}$, provenientes de investigaciones realizadas en el cultivo del ajo en el CIB (Rivera 20II). Se inoculó una porción del tejido fúngico en medio PDA acidificado (ac) con ácido láctico e incubado a $28^{\circ} \mathrm{C}$ por 96 horas.

\section{Pruebas de antagonismo}

Se desarrollaron pruebas de antagonismo usando la metodología de cultivo dual. En el caso de bacteria frente a bacteria, se incubaron cepas de B. subtilis y $P$. marginalis en un medio glucosado. Una placa Petri se rayó hasta la mitad con el patógeno y se colocaron 3 estrías perpendiculares del posible biocontrolador a $2 \mathrm{~cm}$ entre cada una.

Para las pruebas de bacterias contra hongos se empleó agar PDA para evaluar B. subtilis frente a Penicillium sp. y a S. cepivorum; así como para determinar la capacidad antagónica de Trichoderma sp. frente P. marginalis. En estas, las bacterias se rayan en toda la placa y los hongos se colocan en un disco de I cm de diámetro en el centro de ella.
En el caso del hongo frente a hongo, se empleó PDA-ac. para evaluar a Trichoderma sp. contra Penicillium sp. y S. cepivorum. En estas placas se colocó un disco del patógeno y otro del posible biocontrolador en lados opuestos, a $1 \mathrm{~cm}$ del borde (Correa et al. 2007). Las placas se incubaron a $28 \pm 1^{\circ} \mathrm{C}$ y los diámetros de crecimiento del patógeno y del biocontrolador se midieron a las 72, 96 y 120 horas.

Para la evaluación de la capacidad biocontroladora de los microorganismos se empleó la escala utilizada por Ezziyyani et al. (2004a) (Cuadro I) y el cálculo del Porcentaje de Inhibición del crecimiento radial (PICR), que mide el antagonismo de los microorganismos evaluados. Este se obtiene a partir del crecimiento de cada patógeno en cultivo dual, junto con sus respectivos testigos, empleando la fórmula utilizada por Suárez et al. (2008):

$$
P I C R=\frac{R_{1}-R_{2}}{R_{1}} \cdot 100
$$

donde $\mathrm{R}_{1}$ es el radio mayor (radio patógeno-testigo) y $R_{2}$ es el radio menor (radio del patógeno en cultivo dual).

Para las pruebas de bacteria vs bacteria se efectuó el cálculo del potencial antagonista, que consiste en medir el diámetro del halo de inhibición y sustraerlo del diámetro de la colonia del biocontrolador.

\section{Resultados y discusión}

Identificación de aislamientos del género Bacillus

Se determinó que los aislamientos corresponden a B. subtilis, tanto por la morfología de las colonias como por los resultados de las pruebas de identificación del género (figura I, cuadro 2).

Cuadro I. Escala utilizada por Ezziyyani et al. (2004a) para la evaluación de la capacidad antagónica de los biocontroladores.

\begin{tabular}{|c|c|c|}
\hline Grado & Capacidad antagónica & Potencial biocontrolador \\
\hline 0 & Ninguna invasión de la superficie de la cepa patógena & Muy malo \\
\hline 1 & $1 / 4$ Invasión de la superficie de la cepa patógena & Malo \\
\hline 2 & $1 / 2$ invasión de la superficie de la cepa patógena & Deficiente \\
\hline 3 & Total invasión de la superficie de la cepa patógena & Bueno \\
\hline 4 & Total invasión de la superficie de la cepa patógena esporulación sobre ella & Muy bueno \\
\hline
\end{tabular}




\section{Potencial antagónico de Bacillus subtilis}

\section{Bacillus subtilis vs Pseudomonas marginalis}

Los resultados de las pruebas entre $B$. subtilis y $P$. marginalis (figura 2, cuadro 3 ) indican que esta cepa no tiene un buen efecto biocontrolador. La media estadística del potencial antagonista es $1,17 \mathrm{~mm}$, por lo que la cepa de B. subtilis utilizada mostró un porcentaje bajo de poder antibiótico,
Los valores del potencial antagónico a las 120 horas de cultivo, en la prueba de $B$. subtilis vs P. marginalis, presentaron un comportamiento estadístico normal $(P=0,286)$.

\section{Bacillus subtilis vs Sclerotium cepivorum}

Los resultados obtenidos en cultivo dual mostraron que $B$. subtilis no ejerció un buen control sobre el patógeno S. cepivorum (figura 3, cuadro 4). En
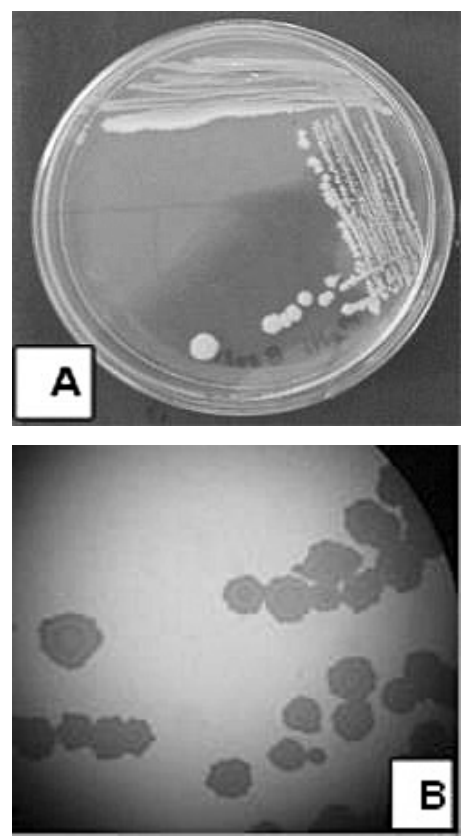

Figura I. Características de las colonias de Bacillus subtilis. A y B) Morfología de colonias de $B$. subtilis, borde aserrado, centro elevado en forma de anillo con consistencia cremosa y pegajosa.
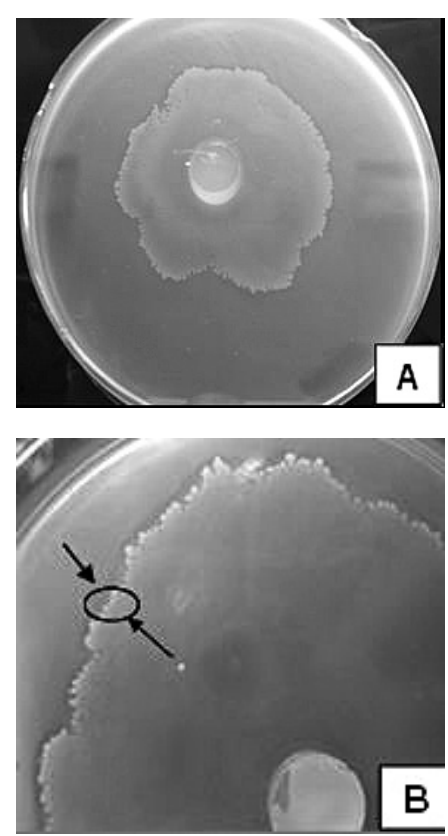

Figura 2. Confrontación de Bacillus subtilis vs Pseudomonas marginalis. A y B) B. subtilis vs $P$. marginalis a las 120 horas de incubación. Se observa la presencia de una pequeña zona clara que separa un microorganismo de otro.

Cuadro 2. Resultados de pruebas de identificación para Bacillus subtilis.

\begin{tabular}{|c|c|c|c|}
\hline Pruebas & $\begin{array}{l}\text { Bacillus } \\
\text { subtilis }\end{array}$ & Pruebas & $\begin{array}{l}\text { Bacillus } \\
\text { subtilis }\end{array}$ \\
\hline Gram & + & Agar Nutritivo I\% dextrosa & + \\
\hline Sangre & + & Voges Proskauer & + \\
\hline Glucosado & + & Citrato Simmonds & + \\
\hline Peptona & + & Tolerancia $6,5 \% \mathrm{NaCl}$ & + \\
\hline Motilidad & + & Hidrólisis Almidón & + \\
\hline Catalasa & + & Rojo de metilo & + \\
\hline Nitrato & + & Oxidasa & + \\
\hline
\end{tabular}

Fuente: Laboratorio de Microbiología, CIB, ITCR 2012. 
los valores del PICR a las 120 horas de cultivo se presentó un comportamiento estadístico normal $(P=0,339)$

Bacillus subtilis vs Penicillium sp.

En la figura 4 y el cuadro 5 se presentan los resultados obtenidos de la medición del antagonismo de

Cuadro 3. Cálculo del potencial antagonista para Bacillus subtilis vs Pseudomonas marginalis.

\begin{tabular}{|c|c|}
\hline Repetición & $\begin{array}{c}\text { Potencial antagonista de Bacillus subtilis vs } \\
\text { Pseudomonas marginalis } \\
(\mathrm{mm})\end{array}$ \\
\hline 1 & 1,24 \\
\hline 2 & 1,11 \\
\hline 3 & 1,40 \\
\hline 4 & 1,12 \\
\hline 5 & 1,10 \\
\hline 6 & 1,28 \\
\hline 7 & 1,03 \\
\hline 8 & 1,14 \\
\hline 9 & 1,08 \\
\hline 10 & 1,26 \\
\hline
\end{tabular}
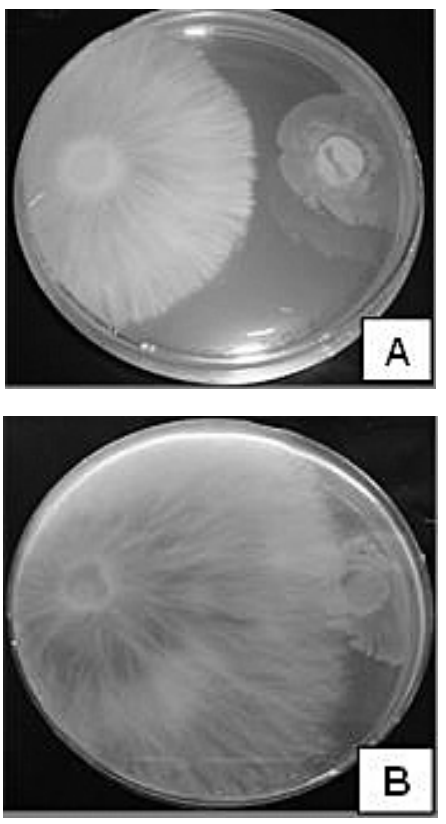

Figura 3. Confrontación de Bacillus subtilis vs Sclerotium cepivorum. A) Prueba a las 72 horas de incubación. B) Prueba a las 120 horas de incubación.
B. subtilis sobre Penicillium sp. En los valores de PICR a las 120 horas de cultivo se evidenció un comportamiento normal $(P=0,85 \mathrm{I})$. Como se puede observar, el biocontrolador no tuvo un buen control sobre el patógeno.

En el cuadro 6 se presenta el resumen de los resultados de B. subtilis frente a los patógenos del ajo evaluados.

Cuadro 4. Cálculo del PICR para Bacillus subtilis vs Sclerotium cepivorum.

\begin{tabular}{|c|c|}
\hline Repetición & $\begin{array}{c}\text { PICR Bacillus subtilis vs Sclerotium } \\
\text { cepivorum }\end{array}$ \\
\hline 1 & 14,22 \\
\hline 2 & 15,56 \\
\hline 3 & 14,82 \\
\hline 4 & 12,70 \\
\hline 5 & 14,62 \\
\hline 6 & 13,99 \\
\hline 7 & 15,89 \\
\hline 8 & 12,20 \\
\hline 9 & 12,28 \\
\hline 10 & 14,59 \\
\hline
\end{tabular}
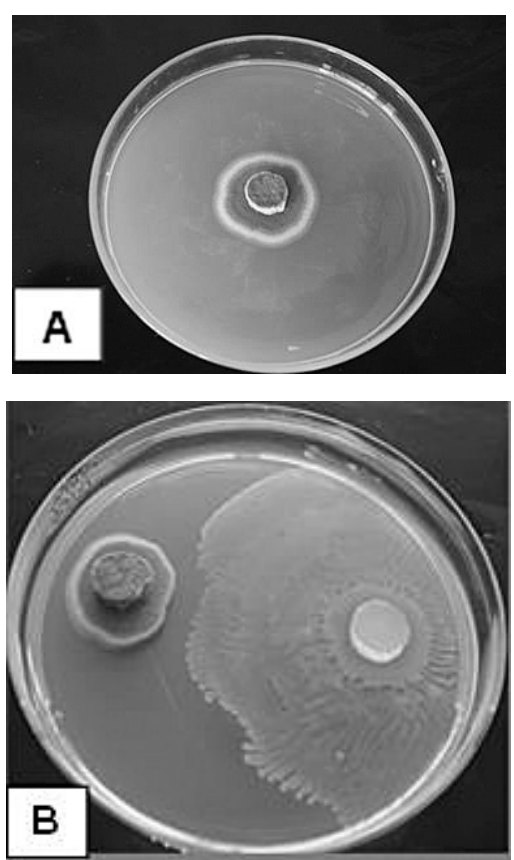

Figura 4. Confrontación de Bacillus subtilis vs Penicillium sp. A) Testigo de Penicillium. B) Prueba a las 120 horas de incubación. 
La media estadística de las pruebas de $B$. subtilis vs S. cepivorum y Penicillium sp. es de 14,087 y 3,328 respectivamente. El análisis de varianza indicó que existen diferencias significativas $(P=0.00)$ en el poder antagonista mostrado por $B$. subtilis frente a los dos patógenos.

La cepa de B. subtilis aislada y evaluada contra cada uno de los patógenos no mostró un buen control de las enfermedades. En especial en los análisis frente a los hongos, el desempeño no fue bueno. Con respecto a su comportamiento frente a la bacteria, aunque el valor de la inhibición es bajo, por las condiciones intrínsecas de la prueba, existe un potencial antagonista que se podría valorar con mayor detalle. Las variaciones en el potencial biocontrolador de una cepa específica están ligadas a las características genéticas y los factores ambientales presentes, así como a las interacciones con otros microorganismos.

Cuadro 5. Cálculo del PICR para Bacillus subtilis vs Penicillium sp.

\begin{tabular}{|c|c|}
\hline Repetición & PICR Bacillus subtilis vs Penicillium sp. \\
\hline 1 & 0,30 \\
\hline 2 & 4,09 \\
\hline 3 & 0,00 \\
\hline 4 & 6,29 \\
\hline 5 & 2,91 \\
\hline 6 & 3,56 \\
\hline 7 & 3,14 \\
\hline 8 & 4,51 \\
\hline 9 & 1,42 \\
\hline 10 & 7,06 \\
\hline
\end{tabular}

Cuadro 6. Resultados del comportamiento de Bacillus subtillis frente a Sclerotium cepivorum y Penicillium sp. según escala de Ezziyyani.

\begin{tabular}{|c|c|c|}
\hline Biocontrolador & Sclerotium cepivorum & Penicillium sp. \\
\hline Bacillus subtilis & Malo & Malo \\
\hline
\end{tabular}

Potencial antagónico de Trichoderma sp.

El método de preservación de la cepa a $-4^{\circ} \mathrm{C}$ fue efectivo y no provocó daños en su integridad. Al cabo de 72 horas a $28 \pm 2^{\circ} \mathrm{C}$, se observó crecimiento activo hasta cubrir por completo la placa en el transcurso de una semana.

\section{Trichoderma sp. vs Pseudomonas marginalis}

En la prueba de antagonismo in vitro de Trichoderma sp. contra P. marginalis se presentó competencia por espacio y nutrientes por parte del biocontrolador, con una zona de inhibición progresiva y continuó creciendo hasta invadir la totalidad de la colonia de la bacteria. Trichoderma sp. evidenció una alta actividad antagónica con un valor de supresión del 32\% (Cuadro 7, Figura 5). En los valores de PICR a las 120 horas de incubación, la prueba de Trichoderma vs $P$. marginalis presenta un comportamiento estadístico normal $(P=0,085)$.

\section{Trichoderma sp. vs Sclerotium cepivorum}

Las pruebas de crecimiento dual de Trichoderma sp. frente a $S$. cepivorum muestran una inhibición progresiva del patógeno a las 120 horas de incubación, lo que coincide con los mecanismos de antibiosis y parasitismo descritos por la literatura (Infante et al. 2009) (figura 6). Según la escala utilizada por Ezziyyani et al. (2004a), Trichoderma sp. obtuvo un grado de 4, que lo califica con muy buen potencial

Cuadro 7. Cálculo del PICR para Trichoderma sp. vs Pseudomonas marginalis.

\begin{tabular}{|c|c|}
\hline Repetición & $\begin{array}{c}\text { PICR. Trichoderma sp. vs Pseudomonas } \\
\text { marginalis }\end{array}$ \\
\hline 1 & 29,55 \\
\hline 2 & 24,47 \\
\hline 3 & 50,92 \\
\hline 4 & 27,12 \\
\hline 5 & 37,88 \\
\hline 6 & 27,19 \\
\hline 7 & 26,53 \\
\hline 8 & 34,71 \\
\hline 9 & 30,43 \\
\hline 10 & 35,15 \\
\hline
\end{tabular}



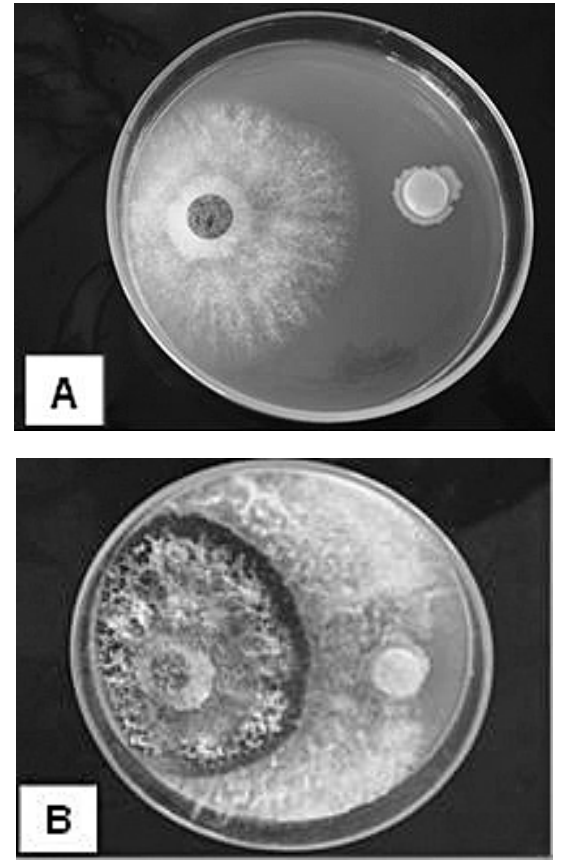

Figura 5. Confrontación de Trichoderma sp. vs Pseudomonas marginalis. A) A las 72 horas. B) A las 96 horas de incubación.
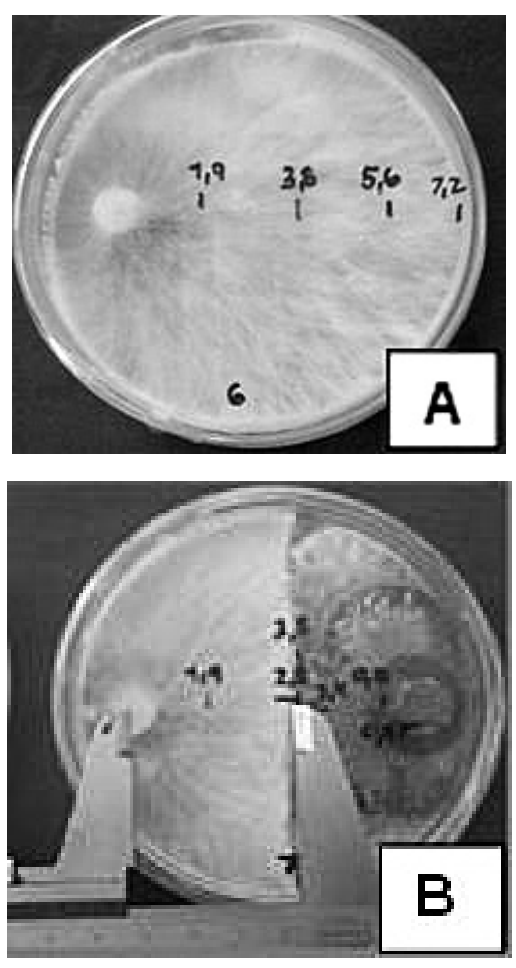

Figura 6. Confrontación de Trichoderma sp. vs Sclerotium cepivorum. A) Testigo de Sclerotium cepivorum. B) Cultivo dual de los dos hongos a las 120 horas. biocontrolador. Lo anterior posiblemente es provocado por metabolitos difusibles y volátiles, así como por enzimas de los grupos celobiohidrolasas, endoglucanasas, glucosidasas, endoquitinasas, endoxilanasas y serinproteasas (Infante et al., 2009).

En el cuadro 8 se presentan los valores de PICR calculados a las 120 horas, donde se demuestra el potencial antagónico del biocontrolador frente a Sclerotium sp. El análisis estadístico de este conjunto de datos mostró que tiene un comportamiento normal $(P=0,257)$.

Trichoderma sp. vs Penicillium sp.

Las pruebas de antagonismo con Trichoderma sp. mostraron inhibición del crecimiento de Penicillium sp. El biocontrolador continuó creciendo hasta invadir totalmente al hongo patógeno al cabo de 120 horas (figura 7). De acuerdo con Harman (2006), este efecto puede estar relacionado con la producción de las enzimas quitinasa, $\beta$-glucanasa, celulasa y proteasa por parte de las cepas de Trichoderma sp.

En los datos de PICR del cultivo dual, la prueba de Trichoderma vs Penicillium presentó un comportamiento estadístico normal $(P=0,254)$ (cuadro 9).

Según la escala utilizada por Ezziyyani et al. (2004a), el hongo Trichoderma sp. obtuvo un valor de 4, que lo califica como muy buen potencial biocontrolador ante Penicillium sp.

Cuadro 8. Cálculo del PICR para Trichoderma sp. vs Sclerotium cepivorum.

\begin{tabular}{|c|c|}
\hline Repetición & $\begin{array}{c}\text { PICR. Trichoderma sp. frente a } \\
\text { Sclerotium cepivorum }\end{array}$ \\
\hline 1 & 49,02 \\
\hline 2 & 46,30 \\
\hline 3 & 42,37 \\
\hline 4 & 37,04 \\
\hline 5 & 35,19 \\
\hline 6 & 37,50 \\
\hline 7 & 39,66 \\
\hline 8 & 20,93 \\
\hline 9 & 45,00 \\
\hline 10 & 49,09 \\
\hline
\end{tabular}



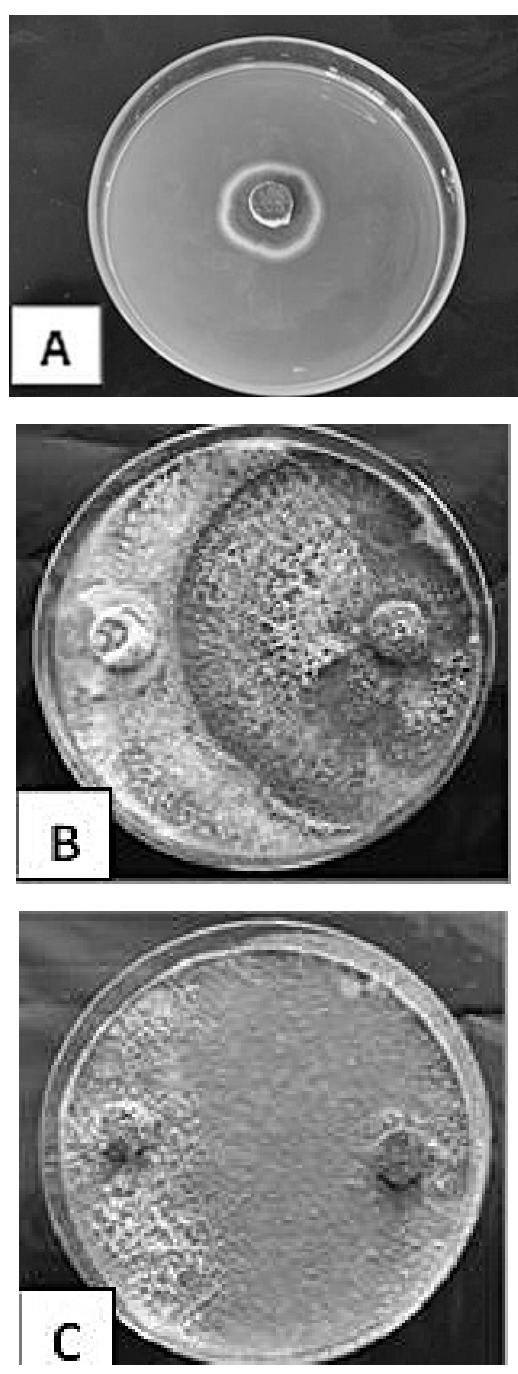

Figura 7. Confrontación de Trichoderma sp. vs Penicillium sp. A) Testigo de Penicillium. B) Prueba a las 96 horas. C) Prueba a las 120 horas.

En el cuadro 10 se presenta el desglose de resultados según el comportamiento de Trichoderma sp. frente a los agentes patógenos.

Trichoderma es un hongo que ataca, parasita y desplaza otros hongos que producen enfermedades en las plantas. Según el análisis de varianza de los porcentajes de inhibición del crecimiento radial como variable se determinó que no existían diferencias significativas entre los tratamientos $(P=0,107)$.

La cepa de Trichoderma sp. utilizada para la prueba antagónica mostró un porcentaje de poder antibió-
Cuadro 9. Cálculo del PICR para Trichoderma sp. vs Penicillium.

\begin{tabular}{|c|c|}
\hline Repetición & PICR Trichoderma sp. vs Penicillium sp \\
\hline 1 & 44,96 \\
\hline 2 & 49,32 \\
\hline 3 & 42,19 \\
\hline 4 & 42,43 \\
\hline 5 & 44,14 \\
\hline 6 & 49,56 \\
\hline 7 & 40,31 \\
\hline 8 & 44,20 \\
\hline 9 & 44,61 \\
\hline 10 & 48,62 \\
\hline
\end{tabular}

Cuadro 10. Resultados del comportamiento de Trichoderma sp. frente a Sclerotium cepivorum y Penicillium sp., según la escala de Ezziyyani.

\begin{tabular}{|c|c|c|}
\hline Biocontrolador & $\begin{array}{l}\text { Sclerotium } \\
\text { cepivorum }\end{array}$ & Penicillium sp. \\
\hline Trichoderma sp. & Muy bueno & Muy bueno \\
\hline
\end{tabular}

tico in vitro muy alto, lo que lo ubica como un buen candidato para el biocontrol de estos patógenos.

El buen desempeño de Trichoderma ante los tres patógenos es un indicador de la alta actividad de los mecanismos de biocontrol disponibles por parte de la cepa. En las pruebas in vitro se obtuvo evidencia de los procesos de competencia, antibiosis y parasitismo que logran la inhibición y destrucción de los patógenos de interés. El grado en que los afecta también está en función de la estructura celular propia de esos patógenos, que permiten el accionar del biocontrolador.

Los resultados obtenidos indican que las cepas estudiadas de Trichoderma sp. pueden utilizarse como antagonistas frente a la pudrición por Penicillium del ajo, bacteriosis por P. marginalis y pudrición blanca por S. cepivorum. No así Bacillus subtilis, pues la cepa aislada no logró controlar los diferentes patógenos. 


\section{Bibliografía}

Astorga, K. (20 I 2). Evaluación in vitro del antagonismo de Bacillus subtilis estirpe silvestre y Trichoderma spp. frente a cepas aisladas e identificadas de Sclerotium cepivorum, Pseudomonas marginalis y Penicillium sp., patógenos de ajo (Allium sativum L.). Trabajo final de graduación Ingeniería en Biotecnología. Instituto Tecnológico de Costa Rica.

Astorga, K, Zúñiga, C. \& Rivera, W. (En prensa). Aislamiento e identificación de patógenos de ajo (Allium sativum L.).

Bergey (2005). Bergey's Manual of Systematic Bacteriology: Volume II: The Proteobacteria. Editorial James T. Staley, Don J. Brenner, Noel R. Krieg. 187 pp.

Brenes Chacón, R., Guillén, A, Hilje, L., Rivera, W. \& Zúñiga, C. (20।3). Cultivo del Ajo en Costa Rica. Editorial UNED. 100 pp.

Claus, D. \& Berkeley, R.C. (1986). Genus Bacillus Cohn 1872 , Bergey's Manual of Systematic Bacteriology. Baltimore, US: Williams y Wilkins.

Correa, S., Mello, M., Ávila, Z., Minare, L., Pádua, R. \& Gomes, D. (2007). Cepas de Trichoderma spp. para el control biológico de Sclerotium rolfsii SAAC. Fitosanidad I I ( ): 3-9.

Ezziyyani, M, Pérez, S.C., Requena, M.E., Rubio, L. \& Candela, M.E. (2004a). Biocontrol por Streptomyces rochei -Ziyani-, de la podredumbre del pimiento (Capsicum annuum L.) causada por Phytophthora capsici. Anales de Biología 26: 69-78. Consultado 20 nov. 2012. Obtenido desde: http://www.um.es/analesdebiologia/numeros/26/PDF/08BIOCONTROL.pdf.

Ezziyyani, M., Pérez, C., Sid Ahmed, A., Requena, M. \& Candela, M. (2004b). Trichoderma harzianum como biofungicida para el biocontrol de Phytophthora capsici en plantas de pimiento (Capsicum annuum L). Anales de Biología 26: 35-45. Consultado 12 jul. 2012. Obtenido desde: http://www.phycoelementa.es/Documents/05-Trichoderma[I].pdf.

Harman, G, (2006). Overview of mechanisms and uses of Trichoderma spp. Phytopathology 96: 190- 194.

Hernández, A., Bautista, S., Velázquez, M. \& Hernández, A. (2007). Uso de microorganismos antagonistas en el control de enfermedades pos cosecha en frutos. Rev Mex Fitopatología 25: 66-74.

Infante, D., Martinez, B., Gonzáles, N. \& Reyes, Y. (2009). Trichoderma mechanisms of action against phytopathogen fungi. Revista de Protección Vegetal 24(I). Consultado 25 sept. 2012. Obtenido desde: http://scielo.sld.cu/scielo. php?script=sci_arttext\&pid=S I 0 | 0-27522009000 100002

Guillén, A., Chacón, R. \& Zúñiga, C. (20119. Detección del virus del enanismo amarillo de la cebolla (OYDV) y el virus latente común del ajo (GCLV) en ajo (Allium sativum L) costarricense. Tecnología en Marcha 24 (4): 47-58.

Izzeddin, N. \& Medina, L. (20 I I). Efecto del control biológico por antagonistas sobre fitopatógenos en vegetales de consumo humano. Salus 15(3). Consultado 28 oct. 2012. Obtenido desde: http://www2.scielo.org.ve/scielo.php?script=sci_ arttext\&pid=S | 3 | 6-7 | 3820 | | $000300005 \&$ lng=es\&nrm=i so.

Lisboa Minguzzi, M.A. (2003). Efectividad de Bacillus subtilis y de una cepa nativa de Trichoderma harzianum sobre la incidencia y severidad de pudrición gris (Botrytis cinérea) en Vid vinifera. Universidad de Talca-Chile, Facultad de Ciencias Agrarias. Escuela de Agronomía (en línea). Consultado 27 jul. 2013. Obtenido desde: http://www.factorhumus.com/ wp-content/uploads/estudios/Control\%20de\%20plagas/ univ.\%20talca.\%20subtilis\%20pudric.\%20gris.pdf.

Reyes, A., Ruiz, E.,Yam, C. \& Dzul, M. (20I I). Selección de Bacillus spp. con actividad antagónica in vitro contra Macrophomina phaseolina (Tassi) en diferentes medios de cultivo. Fitosanidad 15(2): ||7-|2|. Consultado 31 ag. 2012. Obtenido desde: http://www.redalyc.org/articulo.oa?id=209 | 22297007.

Reinoso, Y., Casadesús, L., García, A., Gutiérrez, I., Álvarez, V. \& Álvarez, V. (2006). Aislamiento, selección e identificación de bacterias del género Bacillus antagonistas de Pectobacterium carotovorum. Fitosanidad 10: |87-191. Consultado 28 jul. 2012. Obtenido desde: http://redalyc.uaemex.mx/redalyc/ pdf/209 |/209 | | 610800 |.pdf.

Rivera Méndez, W. (201I). Aislamiento y uso de cepas de Trichoderma spp. Para el control biológico del hongo Sclerotium cepivorum Berkeley, causante de la pudrición blanda de bulbos de ajo (Allium sativum), con miras a la transferencia tecnológica a productores de la zona de Llano Grande de Cartago. Tesis Magister en Gestión de recursos naturales y Tecnologías de producción. Instituto Tecnológico de costa Rica.

Rivera, W., Zúñiga, C. \& Brenes, J. (20/2). Cultivo del ajo. Combate de la enfermedad del torbó (Sclerotium cepivorum). Escuela de Biología. Instituto Tecnológico de Costa Rica. 20 pp.

Rojas, V., Ulacio, D., Sanabria, M.E. \& Jiménez, M.A. (20099. Efecto del calcio, Trichoderma y brócoli en la pared y área celular del ajo para el control de la pudrición blanca. Boletín del Centro de Investigaciones Biológicas 43(2). Consultado 10 sept. 2012. Obtenido desde: http://revistas.luz.edu.ve/index. php/bcib/article/viewFile/340I/3287.

Santander, A. (20/2). Uso de Trichoderma harzianun Rifai y Bacillus subtilis (Ehrenberg) Cohn para el control de (Colletotrichum gloeosporioides Pens.) causante de la antracnosis en mango (Mangifera indica L.) (en línea). Consultado 12 jul. 2012. Obtenido desde: http://saber.ucv.ve/jspui/ handle//23456789/3595.

Suárez, C., Fernández, R.,Valero, N., Gámez, R. \& Páez, A. (2008). Antagonismo in vitro de Trichoderma harzianum Rifai sobre Fusarium solani (Mart.) Sacc. Asociado a la marchitez en maracuyá. Revista Colombiana de Biotecnología I0(3): 35-43. 